\title{
Performance of a Forward Neutron Calorimeter for the ALICE experiment.
}

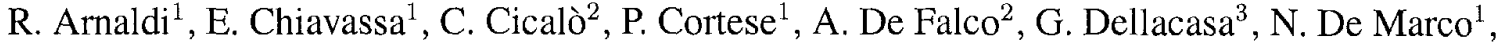

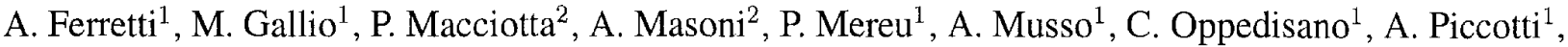 \\ G. Puddu ${ }^{2}$, E. Scalas ${ }^{3}$, E. Scomparin ${ }^{1}$, S. Serci ${ }^{2}$, E. Siddi ${ }^{2}$, G. Usai ${ }^{2}$ and E. Vercellin ${ }^{1}$. \\ For the ALICE Collaboration \\ ${ }^{1}$ Università and I.N.F.N. Sezione di Torino, via P. Giuria 1, 10125 Torino, Italy. \\ ${ }^{2}$ Università and I.N.F.N. Sezione di Cagliari, Cittadella Universitaria, 09042 Monserrato (Ca) , Italy. \\ ${ }^{3}$ Università del Piemonte Orientale, Alessandria, and INFN Sezione di Torino, Italy.
}

The aim of the ALICE experiment [1] is the study of ultrarelativistic heavy ion collisions in which nuclear matter at high temperatures and energy densities can be produced. In these conditions the formation of the state of matter where quarks and gluons are deconfined, i.e. the Quark Gluon Plasma (QGP), could be possible.

ALICE is planned to start taking data in 2005, at the LHC facility at CERN, with two colliding lead ion beams at a c.m. energy of $\sqrt{s}=5.5 \mathrm{TeV}$ per nucleon.

The centrality of the collision is defined by the impact parameter, which can be estimated by measuring the energy carried away by the non-interacting nucleons. The spectator protons and neutrons will be separated from the ion beams, using the separator magnet (D1) of the LHC beam optics [2] and respectively detected by a proton (ZP) and a neutron (ZN) "Zero-degree Calorimeter" (ZDC). An electromagnetic calorimeter is also foreseen for the measurement of the forward photons coming from the decay of $\pi^{0}$, which is anti-correlated with the impact parameter of the collision. It could be used to make the centrality trigger more selective, and less sensitive to possible effects related to the fragmentation of the nuclei. The detectors will be placed at $\sim 115$ meters away from the beam intersection point.

The calorimeters will be placed in a radiation rich environment, of the order of $10^{4} \mathrm{~Gy} /$ day at a luminosity of $2 \cdot 10^{27} \mathrm{~cm}^{-2} \mathrm{~s}^{-1}$. The high interaction rate, corresponding to $\sim 8000$ minimum bias collisions per second, will require a fast detection method. Moreover, since geometrical constraints will limit the lateral dimensions, the transverse size of the detectable shower should be kept as small as possible.

The ZDCs are quartz-fiber calorimeters that exploit the Cherenkov light produced by the shower particles in silica optical fibers [3]. This technique fulfills the above requirements. In addition, quartz-fiber calorimeters are intrinsically insensitive to radio-activation background, which produces particles below the Cherenkov threshold.

The ALICE ZDCs should have an energy resolution comparable with the spectator energy fluctuations, for a given impact parameter, which ranges from $\approx 20 \%$ for central events to $\approx 5 \%$ for peripheral ones, according to simulations that use HIJING as event generator [4]. The fiber-to-absorber filling ratio must be chosen as a compromise between the required energy resolution and the fiber cost.

Two sets of calorimeters will be placed on the opposite sides with respect to the beam intersection point. The proton calorimeters (ZP) will be made of brass as absorber material and its dimensions will be $20.8 \times 12 \times 150 \mathrm{~cm}^{3}$ [5]. Two prototypes of these calorimeters have been already studied [6]. The necessity of containing the shower together with geometrical constraints imposed by the beam pipes, require for the neutron calorimeters (ZN) small transverse dimensions $\left(7 \times 7 \mathrm{~cm}^{2}\right)$ and the use of a dense material.

Two possible solutions are foreseen for the $\mathrm{ZN}$. The calorimeter could be built with the fibers parallel to the beam axis embedded in a tantalum or tungsten alloy passive matrix. A detector of this type has already been built in the NA50 experiment, and its behaviour is well known [7]. However, technical details connected to the cost or the difficulty in machining the materials, suggest the opportunity to test another configuration.

In July 1999, we tested a neutron (ZN) and an electromagnetic calorimeter prototype. Both consist of quartz fiber planes placed at $45^{\circ}$ with respect to the beam axis, sandwiched with tungsten plates. The characteristics of a module are reported in table 1.

Table 1

Characteristics of a prototype module.

\begin{tabular}{|ccc|}
\hline \hline & H Calo (1 mod.) & E.M Calo \\
\hline Dimensions $\left(\mathrm{cm}^{3}\right)$ & $7 \times 7 \times 25$ & $7 \times 7 \times 25$ \\
Filling ratio & $1 / 32$ & $1 / 8$ \\
Absorber material & tungsten & tungsten \\
Number of planes W & 60 & 20 \\
Number of fibers planes & 15 & 19 \\
Fiber material & quartz & quartz \\
Fiber Diameter (core) & $550 \mu \mathrm{m}$ & $550 \mu \mathrm{m}$ \\
Number of PM(XP2020) & 1 & 1 \\
\hline
\end{tabular}

The ZN prototype consisted of two identical modules. In the final configuration it will be made of four modules. The electromagnetic calorimeter consisted of a single module.

To avoid the production of Cherenkov light in the part of the fibers used as optical guides, a system of air guides has been projected and tested. The layout of a module equipped with a light guide is shown in fig. 1 .

The performances of the prototypes were studied by exposing it to the secondary H6 hadron beam at the CERN SPS, at 50, $80,100,120,150,180$ and $200 \mathrm{GeV} / \mathrm{c}$, and to a positron beam of $50,80,100,120,150$ and $200 \mathrm{GeV} / \mathrm{c}$. 


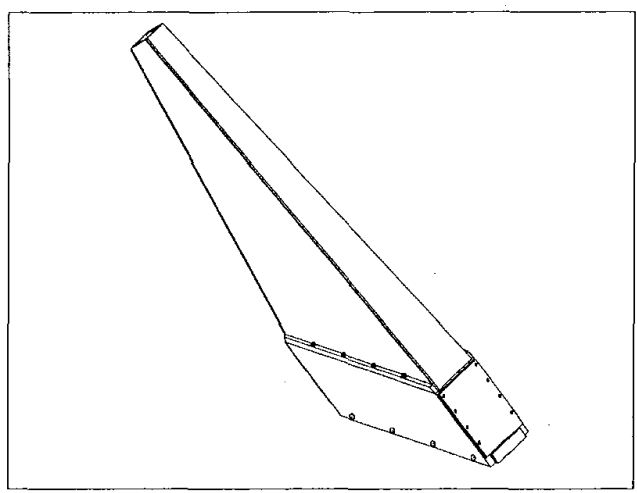

Figure 1: Layout of a calorimeter module.

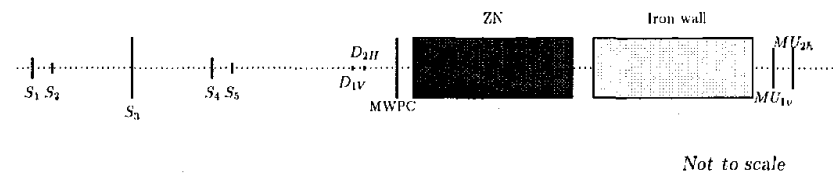

Figure 2: Experimental setup of the test. $\mathrm{S}_{1}, \mathrm{~S}_{2}, \mathrm{~S}_{3}, \mathrm{~S}_{4}, \mathrm{~S}_{5}$ : trigger scintillators. $\mathrm{D}_{1 v}, \mathrm{D}_{2 h}$ : scintillator sticks. $\mathrm{MU}_{1 V}, \mathrm{MU}_{2 H}$ : scintillators for muon detection.

The experimental setup as shown in fig. 2 consists of a plastic scintillator hodoscope, used as trigger system, two small and movable scintillator-sticks used to localize the beam position on the calorimeter surface with an accuracy of $1 \mathrm{~mm}^{2}$, a MWPC installed in front of the calorimeter. The calorimeter itself is placed on a movable platform; two plastic scintillation counters beyond an iron wall detect muons.

Several air-core light guides were tested. Four passive plates $2.5 \mathrm{~mm}$ thick, followed by other four plates equipped with fibers could be displaced at different $\mathrm{z}$ positions inside a dedicated module (fig. 3). This module allowed us to measure the uniformity of the light transmission as a function of $z$, when irradiating it with a $100 \mathrm{GeV}$ positron beam. The uniformity in the $\mathrm{x}$ direction was studied by moving it in the left or right side with respect to the beam.

We used two reflecting guides $80 \mathrm{~cm}$ and $50 \mathrm{~cm}$ long (GL80 and GL50), and a diffusing guide of $50 \mathrm{~cm}$ (GB50).

The former shows a higher and more uniform response (within 15\%) as a function of $\mathrm{z}$ (fig. 4). It also shows the best resolution. Thus, we used the GL80 guide for the subsequent tests.

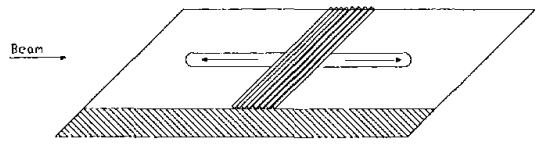

Figure 3: Layout of the module used to test the guides.

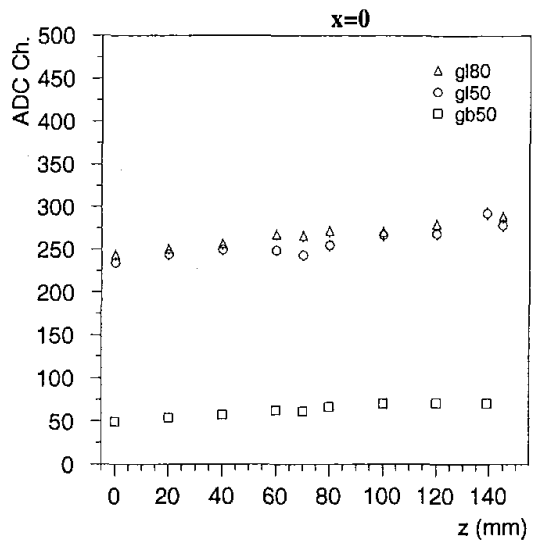

Figure 4: Response of three light guides as a function of $z$ with a positron beam centered at $x=0$.

In fig. 5 the linearity of the response as a function of the hadron beam energy is shown for ZN. The trend is linear also for positrons.

The resolution was also determined. However, it has to be stressed that the result is affected by the fact that the shower is not fully contained in the longitudinal direction. The energy resolution ranges from $\sim 70 \%$ to $\sim 30 \%$, and has been fitted with the formula $R=490 / \sqrt{E}$. In the final version the calorimeter will be made of four modules, thus assuring a better containment.

In order to evaluate the hadronic shower transverse size, we studied the response of the calorimeter as a function of the horizontal beam impact coordinate.

The information about the beam position is given by the MWPC (fig. 6 UP). In the same figure (down) we show the derivative of the previous distribution, from which one can estimate the transverse shower size. The FWHM is $4.5 \pm 0.4 \mathrm{~mm}$.

The tests performed with the E.M. calorimeter concern mainly the linearity and the resolution, which are shown in fig. 7,8.

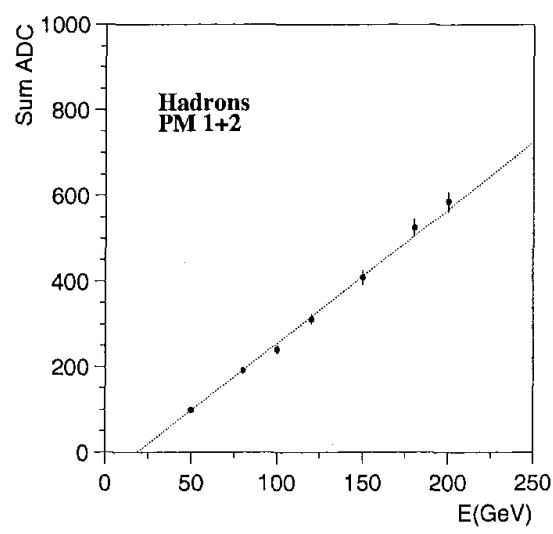

Figure 5: Linearity of the response as a function of the energy for an hadron beam.

From the test beam measurements, the $80 \mathrm{~cm}$ long, reflecting 

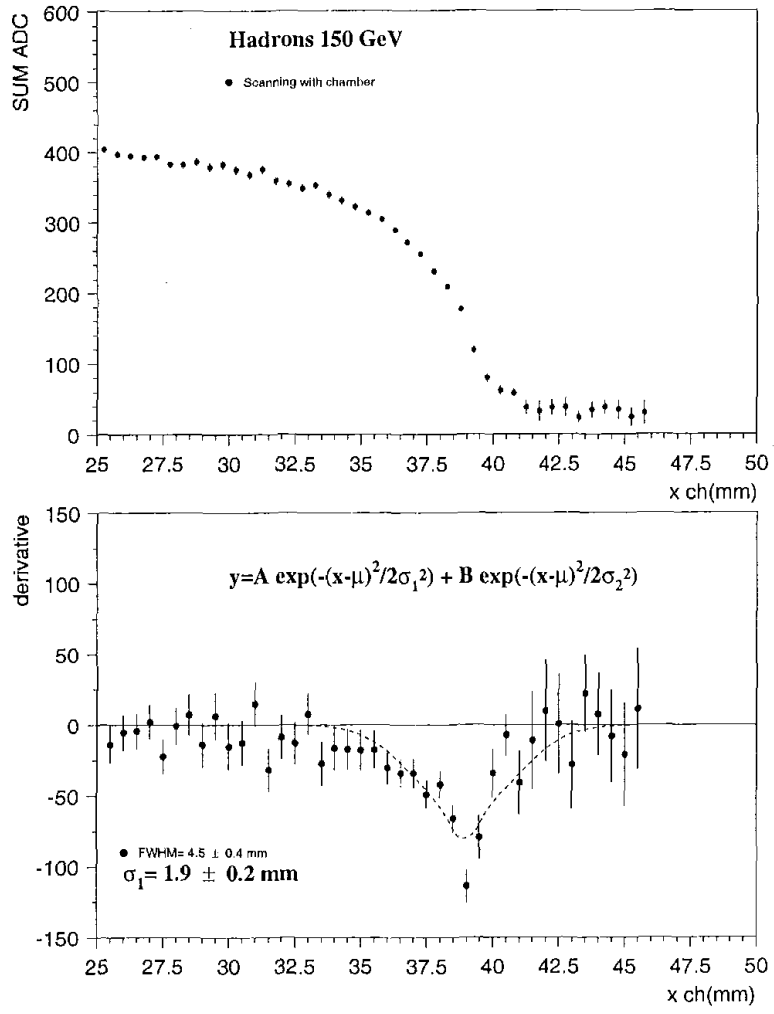

Figure 6: Up: response of the calorimeter as a function of the beam impact coordinate. Down: derivative of the response.

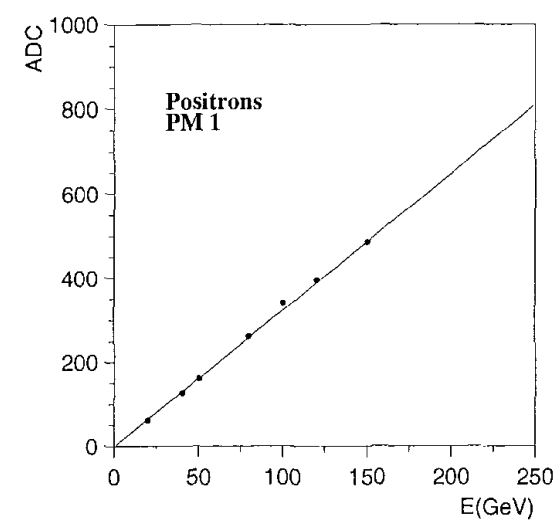

Figure 7: Linearity of the response for the E.M. calorimeter.

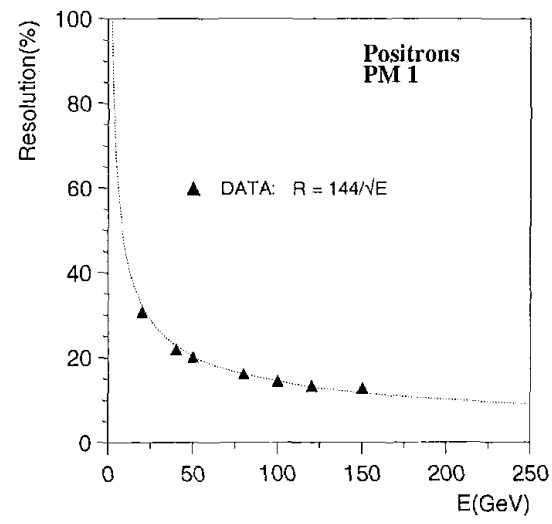

Figure 8: Energy resolution for the E.M. calorimeter.

air guide is to be preferred to the other ones. The results for the resolution of the $\mathrm{ZN}$ prototype are not the final ones. A new test with a full detector is foreseen in year 2000 .

We wish to thank all the people that contributed to the design, construction and successful operation of the calorimeters. In particular the technical support provided by M. Arba and M. Tuveri from INFN Cagliari and G. Alfarone from INFN Torino. The help and suggestions of K. Elsener during the test with hadrons and positrons were greatly appreciated.

\section{REFERENCES}

[1] "ALICE Technical Proposal" CERN/LHCC 95-7I.

[2] "LHC Conceptual Design" CERN/AC/95-05 (LHC).

[3] P.Gorodetsky et al., NIM, A 361, (1995) 161.

[4] X.N.Wang and M.Gyulassy, Phys. Rev., D 44, (1991) 3501.

[5] "ALICE ZDC Technical Design Report" CERN/LHCC 99. 5.

[6] "Performance of a forward hadron calorimeter for the ALICE experiment" (to be published in the IEEE98 NFS proceedings.

[7] R. Arnaldi et al., NIM, A 4II (1998) 1. 